\title{
Accurate Sparse-Projection Image Reconstruction via Nonlocal TV Regularization
}

\author{
Yi Zhang, Weihua Zhang, and Jiliu Zhou \\ College of Computer Science, Sichuan University, No. 24, South Section 1, Yihuan Road, Chengdu 610065, China \\ Correspondence should be addressed to Weihua Zhang; zhangweihua@scu.edu.cn
}

Received 26 August 2013; Accepted 24 November 2013; Published 27 January 2014

Academic Editors: C.-J. Lu, Z. Shi, and P. Sosik

Copyright (C) 2014 Yi Zhang et al. This is an open access article distributed under the Creative Commons Attribution License, which permits unrestricted use, distribution, and reproduction in any medium, provided the original work is properly cited.

\begin{abstract}
Sparse-projection image reconstruction is a useful approach to lower the radiation dose; however, the incompleteness of projection data will cause degeneration of imaging quality. As a typical compressive sensing method, total variation has obtained great attention on this problem. Suffering from the theoretical imperfection, total variation will produce blocky effect on smooth regions and blur edges. To overcome this problem, in this paper, we introduce the nonlocal total variation into sparse-projection image reconstruction and formulate the minimization problem with new nonlocal total variation norm. The qualitative and quantitative analyses of numerical as well as clinical results demonstrate the validity of the proposed method. Comparing to other existing methods, our method more efficiently suppresses artifacts caused by low-rank reconstruction and reserves structure information better.
\end{abstract}

\section{Introduction}

Computed tomography (CT) has still been a widely used medical imaging technology for clinical diagnosis. However, according to the recent reports, the risk of overhigh radiation has caused social attention. It is well known that it is harmful for human body to expose to heavy radioactive source. As a result, the problem which arises is, when CT scans are inevitable, and how can we reduce the radiation dose without losing the imaging quality?

To deal with this issue, many technologies which can be categorized into two groups were proposed. The first one is to lower the configuration parameters of X-ray. The key step is to reduce the milliampere seconds ( $\mathrm{mAs}$ ) or $\mathrm{kVp}$ parameter; however, the quantum noises also appear. Many methods were proposed to suppress the quantum noises [1-6]. The vital problem of this kind is that under the situation of low operational current or voltage, when high density objects, such as metal implants or bones exit, the severe attenuation of X-rays allows only a limited number of photons to reach detectors. As a result, new artifacts will be introduced in the reconstructed image. The artifacts spread through the whole image, which contaminate the imaging quality. Therefore, the second category does not change the energy of X-ray. Instead of that, reducing the projection number which is also called sparse-projection reconstruction is another way to achieve this goal. However, due to the lack of projection views, streak artifacts will severely affect the imaging quality. This topic can be viewed as an ill-posed inverse problem which has provoked many studies about it. In this paper, we will focus on sparse-projection reconstruction.

Compressive sensing (CS) is an efficient method to handle sparse-projection reconstruction $[7,8]$. The main idea of CS is very similar to sparse-projection reconstruction and both of them manage to recover the complete signals from a severe undersampling. Many studies have been done following such concepts. In [9], Chen et al. considered a prior image as a prior knowledge (PICCS). Based on the fact that in many CT imaging applications some physical and anatomical structures and the corresponding attenuation information of the scanned object can be a priori known, Rashed and Kudo presented a statistical iterative reconstruction (SIR) by incorporating this prior information into the image reconstruction objective function [10]. Ma et al. introduced nonlocal means into low-dose reconstruction with a precontrast scan [11]. The most famous model with CS theory is total variation (TV) based method called ASD-POCS which is firstly proposed by Sidky et al. $[12,13]$. They introduced TV into algebraic 
reconstruction technique (ART) to suppress the artifacts caused by the limitation of projection views. Following this idea, the same group replaced $L_{1}$ norm with $L_{p}$ norm in the minimization function [14]. Ritschl et al. proposed a step-size-adaptive method based on TV to eliminate the dependence on the raw data consistency [15]. To improve the convergence and efficiency of TV based minimization methods, Yu and Wang constructed a pseudoinverse of discrete gradient transform (DGT) and adapted a softthreshold filtering algorithm [16]. Lu et al. proposed a novel algorithm for image reconstruction from few-view data. It utilizes the simultaneous algebraic reconstruction technique (SART) coupled with dictionary learning, sparse representation, and TV minimization on two interconnected levels [17]. Although TV based methods have achieved efficient results, TV suffered from the notorious blocky effect which obstructs the clinical practice of TV. To overcome this disadvantage; many efforts were made [18-20]. Zhang et al. combined classical TV with a high-order norm to suppress the blocky effect [21]. Fractional calculus was introduced into ASDPOCS model [22]. By adjusting the order of fractional-order TV norm, blocky effect can be reduced to an acceptable level without increasing much computational cost. The reason of this side effect should put the blame on the basic assumption of TV that the images are piecewise constant and TV is a local-related computation.

In this paper, we propose a nonlocal TV based model to deal with the sparse-projection image reconstruction. First, we will review the classical TV based model in the next section. The details of our method are represented in Section 3. Numerical and clinical experiment results are demonstrated in Section 4. The discussion and conclusion are given at the end of this paper.

\section{A Brief Review about TV Based Image Reconstruction Method}

Because our method is an extended version of TV based image reconstruction, in this section, we first give a brief description of this method. Given a 2-dimensional image $u=u(\mathbf{x})=u_{x_{1}, x_{2}}$, whose size is $M \times N, x_{1} \in[1, M]$ and $x_{2} \in[1, N]$, for any $u$, the gradient operator is defined as

$$
\nabla u=\left(\Delta_{x_{1}} u, \Delta_{x_{2}} u\right),
$$

where $\Delta_{x_{1}}$ and $\Delta_{x_{2}}$ are the first-order differential operators along $x$-axis and $y$-axis, respectively. $\Delta_{x_{1}}$ and $\Delta_{x_{2}}$ can be represented as

$$
\begin{aligned}
& \Delta_{x_{1}} u=u_{x_{1}, x_{2}}-u_{x_{1}-1, x_{2}}, \\
& \Delta_{x_{2}} u=u_{x_{1}, x_{2}}-u_{x_{1}, x_{2}-1} .
\end{aligned}
$$

In traditional CT imaging problem, the sampling procedure can be considered as a discrete linear transform

$$
A u=f,
$$

where $A$ is the system matrix which is comprised of $I$ row vectors and $f=\left(f_{1}, f_{2}, \ldots, f_{I}\right)^{T}$ is the measurement vector.

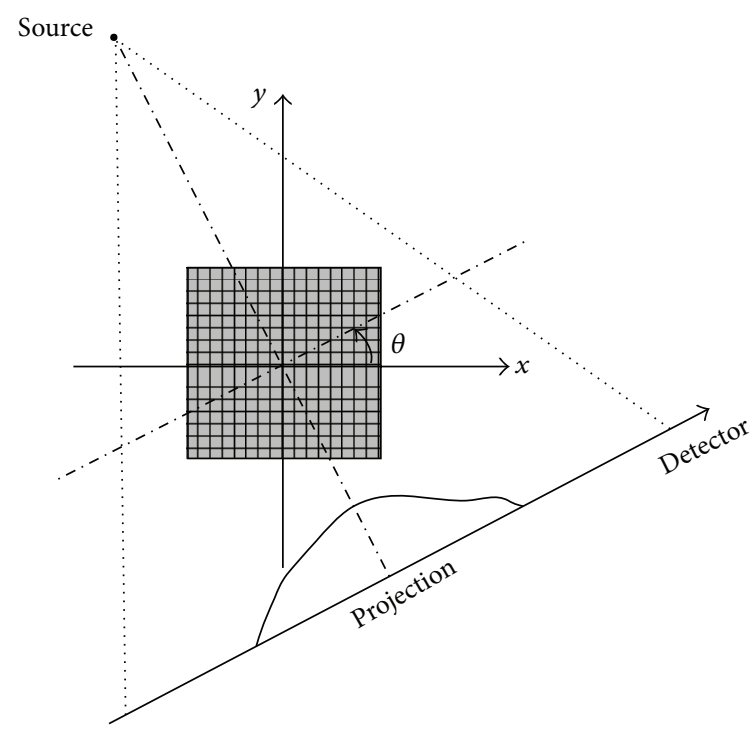

FIgURE 1: Fan-beam CT geometry configuration.

The individual elements of the system matrix are $A_{i j}$, where $i=1,2, \ldots, I$ and $j=1,2, \ldots, J$. It is obvious that $M \times N=J$. Without losing generality, the fan-beam projection geometry can be demonstrated in Figure 1.

To solve the linear system in (3), the TV based image reconstruction algorithm which was used to deal with the sparse-projection limitation is to optimize the following problem $[12,13]$ :

$$
\min \|u\|_{\mathrm{TV}} \quad \text { subject to } u \geq 0, \quad A u=f,
$$

where $\|u\|_{\mathrm{TV}}$ can be considered as a $L_{1}$ norm of the firstorder gradient image $\nabla u$. The TV based algorithm combined the steepest decent method and the projection on convex sets (POCS) to achieve the solution of (4) iteratively.

\section{The Proposed Nonlocal TV Reconstruction Method}

The locality of TV is the main factor which causes blocky effect and it is the motivation we introduce nonlocal TV based method for alleviating this phenomenon. The minimization problem of CT image reconstruction can be formulated as

$$
\min J(u) \quad \text { subject to } u \geq 0, \quad A u=f,
$$

where $J(u)$ is the regularization term and other symbols are with same meanings as (4). The key part is how to choose $J(u)$. In TV based model, $J(u)=J_{\mathrm{TV}}(u)=\|u\|_{\mathrm{TV}}$ and in our proposed method, $J(u)=J_{\text {NLTV }}(u)=\|u\|_{\text {NLTV }}$. Inspired by [23-26], we define $\|u\|_{\text {NLTV }}$ as

$$
\|u\|_{\mathrm{NLTV}}=\int_{\Omega}\left|\nabla_{\mathrm{NLTV}} u(\mathbf{x})\right| d \mathbf{x},
$$

where the nonlocal gradient $\nabla_{\text {NLTV }} u(\mathbf{x})$ is defined as the vector of all partial differences $\nabla_{\mathrm{NLTV}} u(\mathbf{x}, \cdot)$ at $\mathbf{x}$ such that

$$
\nabla_{\mathrm{NLTV}} u(\mathbf{x}, \mathbf{y})=(u(\mathbf{y})-u(\mathbf{x})) \sqrt{w(\mathbf{x}, \mathbf{y})}, \quad \forall \mathbf{y} \in \Omega .
$$


So we obtain the minimization function

$$
\begin{aligned}
& \min \int_{\Omega} \sqrt{\int_{\Omega}(u(\mathbf{x})-u(\mathbf{y}))^{2} w(\mathbf{x}, \mathbf{y}) d \mathbf{y}} d \mathbf{x} \\
& \text { subject to } \quad u \geq 0, \quad A u=f
\end{aligned}
$$

where $w(\mathbf{x}, \mathbf{y})$ is a weighting function to compute the similarity between vectors $\mathbf{x}$ and $\mathbf{y} . w(\mathbf{x}, \mathbf{y})$ is defined as [25]

$$
w(\mathbf{x}, \mathbf{y})=\exp \left(-\frac{G_{\alpha} *|u(n(\mathbf{x}))-u(n(\mathbf{y}))|^{2}}{h^{2}}\right)
$$

where $G_{\alpha}$ is the Gaussian kernel with standard deviation $\alpha$ and $h$ is filtering parameter controlling the decay of the exponential function. Generally, $h$ is determined by the noise level. $n(\mathbf{x})$ and $n(\mathbf{y})$ denote the two local similarity neighborhoods (named patch windows) centered at the pixels $\mathbf{x}$ and $\mathbf{y}$, respectively, and we only compute the weights in a semilocal searching window for each pixel. To simplify the optimization problem, we reformulate (5) as

$$
E(u)=\int_{\Omega}\left|\nabla_{\mathrm{NLTV}} u(\mathbf{x})\right| d \mathbf{x}+\frac{\lambda}{2}\|A u-f\|_{2}^{2},
$$

where $\lambda$ is a parameter to control the balance between regularization and fidelity terms.

Then, we use the gradient descent to update the solution by the Euler-Lagrange equation of (10):

$$
u_{t}=-R u+\lambda A^{*}(f-A u),
$$

where $A^{*}$ is the adjoint of $A$ and

$$
\begin{aligned}
& R u \\
& =-\int_{\Omega}(u(\mathbf{y})-u(\mathbf{x})) w(\mathbf{x}, \mathbf{y}) \\
& \quad \times\left[\frac{1}{\left|\nabla_{\mathrm{NLTV}} u(\mathbf{x})\right|}+\frac{1}{\left|\nabla_{\mathrm{NLTV}} u(\mathbf{y})\right|}\right] d \mathbf{y}
\end{aligned}
$$

Our proposed method for sparse-projection image reconstruction can be summarized by the following steps:

(a) initialization of performance parameters and stopping criteria;

(b) outer loop for $p=1,2, \ldots, P$ : reconstruction with SART algorithm:

$$
\begin{gathered}
u_{j}^{p+1}=u_{j}^{p}+\frac{1}{A_{+, j}} \sum_{i=1}^{I} \frac{A_{i, j}}{A_{i,+}}\left(f_{i}^{0}-f_{i}^{p}\right), \\
A_{i,+}=\sum_{j=1}^{J} A_{i, j}, \quad \text { for } i=1, \ldots, I, \\
A_{+, j}=\sum_{i=1}^{I} A_{i, j}, \quad \text { for } j=1, \ldots, J ;
\end{gathered}
$$

(c) inner loop for $q=1,2, \ldots, Q$ : NLTV gradient descent method:

$$
{ }_{q+1} u=-R_{q} u+\lambda A^{*}\left(f-f^{p}\right) ;
$$

(d) repeat beginning with step (b) until the stopping criteria are satisfied.

\section{Numerical and Clinical Results}

In this section, to validate and evaluate the proposed method, numerical and clinical experiments are performed. In the numerical experiments, Shepp-Logan phantom was used and we simulated X-ray projections using Siddon's ray-driven algorithm [27] in fan-beam geometry. The source to rotation center distance is $40 \mathrm{~cm}$ and the detector to rotation center is $40 \mathrm{~cm}$. The image array is $20 \times 20 \mathrm{~cm}^{2}$. The detector whose length is $41.3 \mathrm{~cm}$ is modeled as a straight-line array of 512 detector bins. All the tests are performed by MATLAB on a PC with Intel i7-3770 CPU $3.40 \mathrm{GHz}$ and $8 \mathrm{~Gb}$ RAM. In clinical experiment, we applied our method to a typical CT slice of a human chest. All the scans were performed on a Siemens SOMATOM Sensation 64 MSCT scanner (Siemens Medical System, Erlangen, Germany) except for the SheppLogan (S-L) phantom. The voltage and current were $120 \mathrm{kVp}$ and $200 \mathrm{~mA}$ with a slice thickness of $1 \mathrm{~mm}$. To get a good visual effect of our method, we compare the proposed method to FBP, EM, and ASD-POCS. The reconstruction results are also quantitatively evaluated in terms of RMSE and MSSIM whose computational definitions are given in $[28,29]$. In all the experiment results, the main parameters were set as $\alpha=$ 1 , the search window size $N_{s}=5 \times 5$, the patch window size $N_{p}=21 \times 21, \lambda=0.1, P=100$, and $Q=20$. Moreover, we chose $h$ to be the estimated noise variance in the filtered back projection image. We used a wavelet based noise estimation model introduced by Donoho and Johnstone in these experiments [30].

4.1. Phantom Cases. In this section, the numerical experiment results are given. The results were performed under ideal condition, in which projection data were generated numerically without adding noise. To demonstrate the performance of our method, Shepp-Logan phantom was uniformly sampled with 30 over 360 degrees. The iteration numbers of EM, ASD-POCS, and NLTV were simply set to 100 .

The reconstruction results of Shepp-Logan phantom are given in Figure 2. In Figure 2(b), it is obvious that, due to incompleteness of projection data, classical FBP cannot achieve an acceptable solution. Although EM is a widely used method, its result in Figure 2(c) is not satisfactory. The whole image is fulfilled with severe artifacts and it is difficult to recognize any parts of the phantom. In Figures 2(d) and 2(e), ASD-POCS and NLTV suppress most of the artifacts. ASDPOCS recovers all the virtual organs except some structural parts. As we marked with white arrows in Figures 2(d) and 2(e), when ASD-POCS dealt with regions full of small structure information, oversmoothing effect appeared. Different parts with very small intervals are obscure and some edges are 


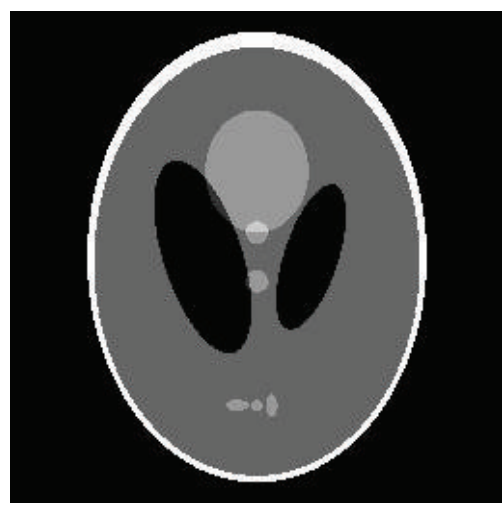

(a)

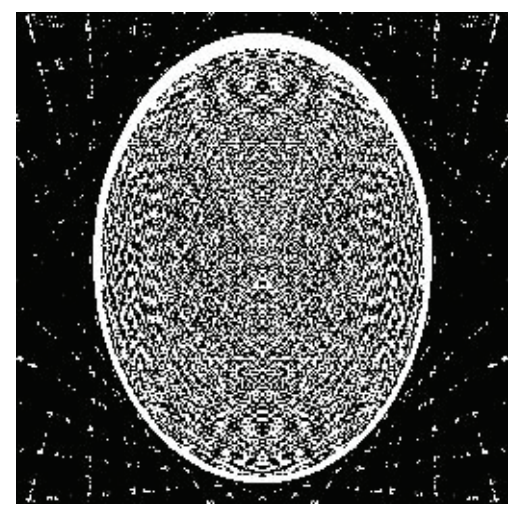

(b)

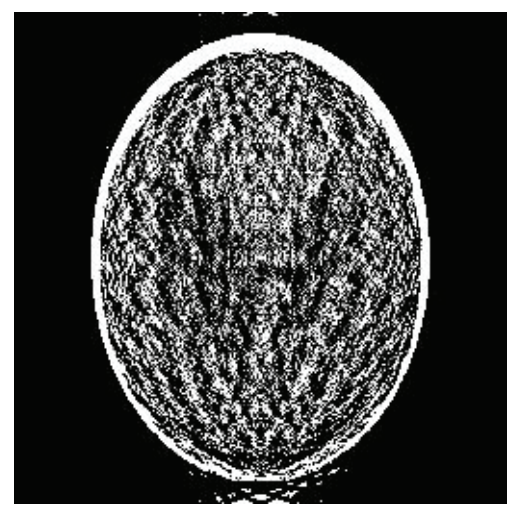

(c)

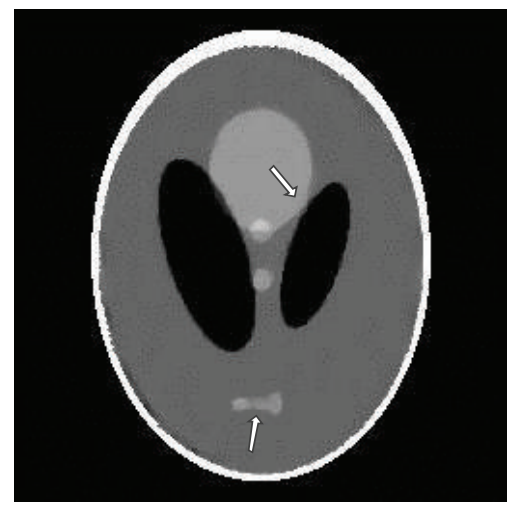

(d)

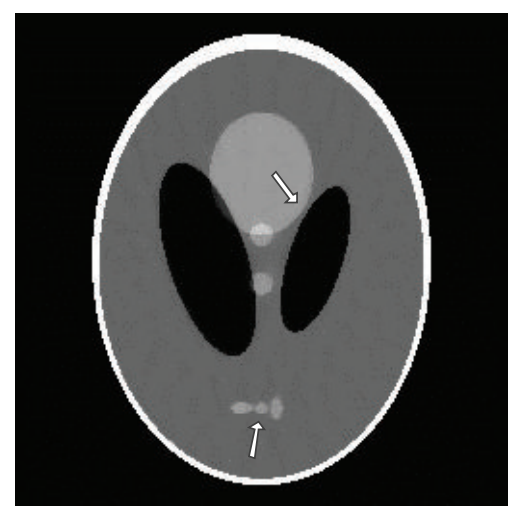

(e)

Figure 2: Reconstruction results of Shepp-Logan phantom by different methods. (a) Original, (b) FBP, (c) EM, (d) ASD-POCS, and (e) NLTV.

TABLE 1: Evaluations of numerical phantom reconstruction.

\begin{tabular}{lcccc}
\hline & RMSE & MSSIM & Iterations & Time (s) \\
\hline FBP & 0.4073 & 0.4568 & - & $\mathbf{0 . 0 2 9 0}$ \\
EM & 0.1548 & 0.7896 & 100 & 2.3878 \\
ASD-POCS & 0.0062 & 0.9932 & 100 & 5.9048 \\
NLTV & $\mathbf{0 . 0 0 2 2}$ & $\mathbf{0 . 9 9 7 6}$ & 100 & 69.87 \\
\hline
\end{tabular}

also blurred. Compared to ASD-POCS, NLTV can reduce the impact to a certain extent. The edges in Figure 2(e) are well kept and three oval organs in the bottom of image are more distinguishable. Meanwhile, the corresponding quantitative evaluations are shown in Table 1. Statistically, NLTV yields better RMSE and MSSIM than those of other methods, but the computational cost is much higher.

4.2. Clinical Cases. In this section, we validated the proposed method on a clinical case which was scanned by a Siemens SOMATOM Sensation 64 MSCT scanner (Siemens Medical System, Erlangen, Germany). The voltage and current were $120 \mathrm{kVp}$ and $200 \mathrm{~mA}$ with a slice thickness of $1 \mathrm{~mm}$. To demonstrate the effectiveness of our method, the results processed by FBP, EM, and ASD-POCS are given for comparison. The full scanned image with 550 views is used as a reference image. The image is downsampled uniformly to 20 views, about two-tenths of the original dataset.
The iteration numbers of EM, ASD-POCS, and NLTV were set to 100 uniformly. The reconstructed images are displayed in Figure 3.

It is obvious that FBP and EM cause considerable streaklike artifacts in Figures 3(b) and 3(c) and the structure information of tissues is of terrible vision. ASD-POCS and NLTV dispel most of the artifacts, so that most of the tissues can be seen in Figures 3(d) and 3(e). However, in Figure 3(d), the boundaries between different tissues are blurred and the blocky effects are visible in the tissues. Three examples are given in Figures 3(d) and 3(e) by white arrows. NLTV kept most of structure information well and much less side effect. The zoomed parts indicated by white arrows are displayed in Figure 3(f). The quantitative evaluations are shown in Table 2. It can be seen that the results show the coherence with the results of numerical phantom. NLTV obtains better RMSE and MSSIM than other methods but suffers from larger computation overheads.

\section{Discussion and Conclusion}

With the development of modern medical imaging technologies, CT has been playing an increasing important role in clinical analysis. Image reconstruction with sparse projection is one of the most efficient ways to lower the dose the patients will endure. CS is a powerful tool to deal with this problem. CS has proved that a complete signal can 


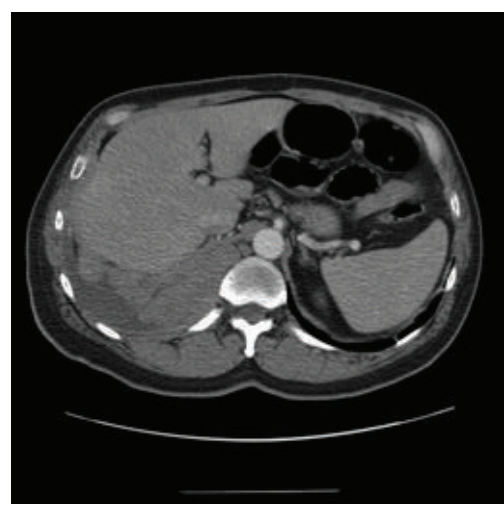

(a)

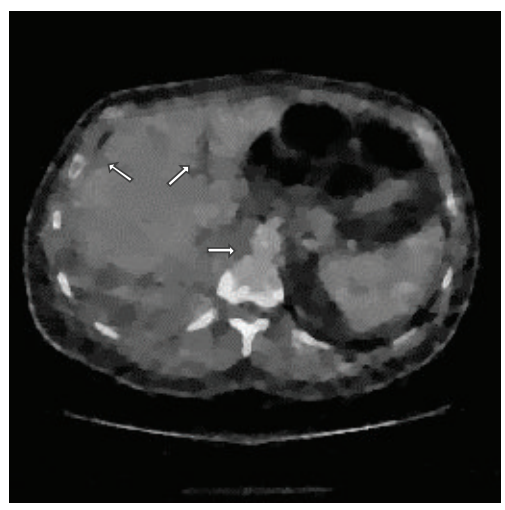

(d)

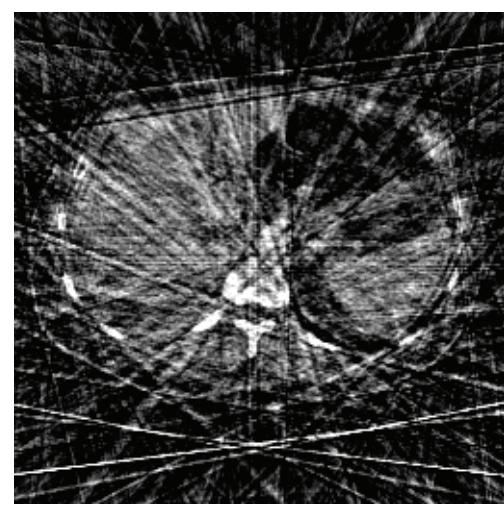

(b)

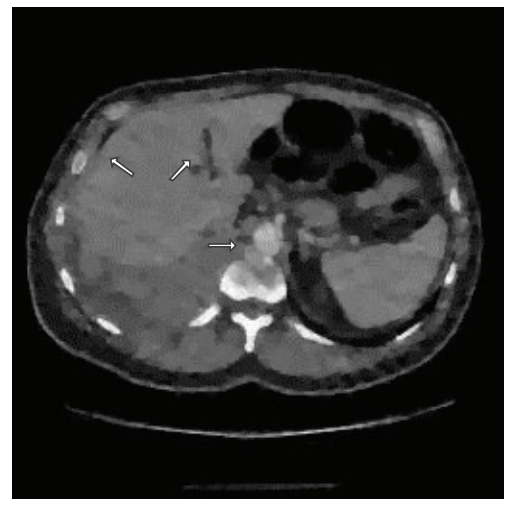

(e)

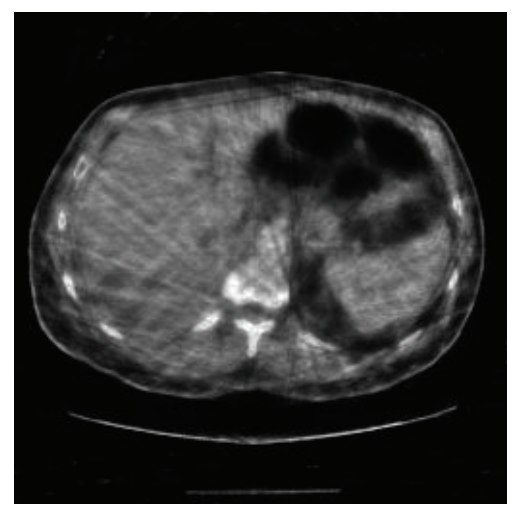

(c)
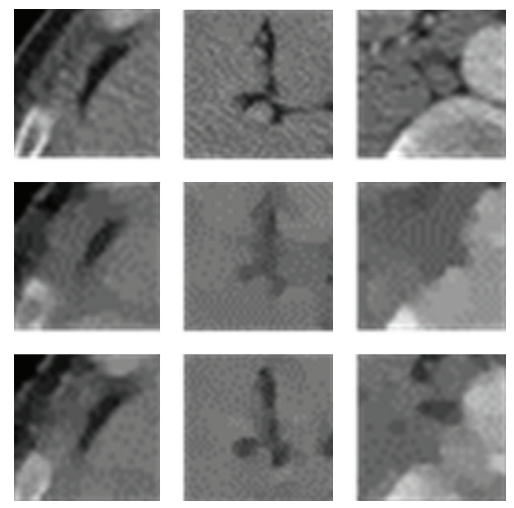

(f)

FIGURE 3: Reconstruction results of chest image by different methods. (a) Original, (b) FBP, (c) EM, (d) ASD-POCS, (e) NLTV, and (f) zoomed parts of original image, ASD-POCS, and NLTV. First row is original images, the second row is ASD-POCS results, and the third row is NLTV results.

TABLE 2: Evaluations of clinical image reconstruction.

\begin{tabular}{lcccc}
\hline & RMSE & MSSIM & Iterations & Time $(\mathrm{s})$ \\
\hline FBP & 0.2260 & 0.6779 & - & $\mathbf{0 . 0 3 1 0}$ \\
EM & 0.0581 & 0.7190 & 100 & 2.9973 \\
ASD-POCS & 0.0562 & 0.7371 & 100 & 6.1286 \\
NLTV & $\mathbf{0 . 0 4 0 3}$ & $\mathbf{0 . 8 2 1 4}$ & 100 & 84.53 \\
\hline
\end{tabular}

be recovered, while a sparsifying transform exists. In this situation, Nyquist sampling theory may not be fit. TV is widely used as an efficient sparsifying transform and it can be introduced into many topics in CT reconstruction, such as sparse projection, limited-angle, and interior reconstruction. Although TV is popular, the blocky effect in homogeneous regions limits the applications in clinical practice. The main reason for this phenomenon is that TV is neighborhood based and there is no global information involved. This will lead to loss of structure and texture information. To solve this problem, we introduce NLTV into medical imaging and give its application in sparse-projection reconstruction. NLTV calculates the weights by searching in all the image patches and it avoids being dependent only on neighboring pixels. The experimental results show that the presented NLTV method can yield more significant performance gains than the existing methods, including FBP, EM, and ASD-POCS, in terms of visual effect and different measurement metrics.

There are several parameters in our methods. All of them should be determined manually, namely, the search window size $N_{s}$, the patch window size $N_{p}$, the filtering parameters $\alpha$, and the regularization scale parameter $\lambda$. Note that, all the parameters are application related. In our purpose, a bigger $N_{s}$ theoretically means that more similarity information will be acquired. By extensive experiments, $N_{s}=$ $21 \times 21$ and $N_{p}=5 \times 5$ will be appropriate settings for effective noise and artifact suppression while maintaining computational efficiency. For the other parameters, $\alpha$ and $\lambda$, in our simulations, we simply select the best average configuration based on the results obtained with a broad range of parameter values manually in terms of visual inspection and quantitative measurements. Due to the computational cost of the proposed method, an adaptive mechanism will be useful and in the future work, we will focus on this problem.

Another concern is the computation cost of the proposed method. As a result of introducing global patch distance computation, the computational burden is much bigger than other iteration based methods. However, with the rapid development of storage hardware, the processing time will not be main obstacle and also the proposed method can be implemented on PC clusters or on graphic processing units (GPU), which will make it feasible for practical application. 
In conclusion, in this paper, we present a novel sparseprojection image reconstruction method using nonlocal total variation. After experiments on numerical phantoms and clinical cases, the proposed method shows better performance than several commonly used methods with respect to both quantitativeness and qualitativeness. Although the computational cost of this method is larger than current methods, there are several methods that can accelerate the processing speed. It will be convenient to implement and add to modern CT systems. The optimization of adaptive parameter selection and acceleration is another concern in our future work.

\section{Conflict of Interests}

The authors declare that there is no conflict of interests regarding the publication of this paper.

\section{Acknowledgments}

This work is supported by the grants from the National Science Foundation of China (nos. 61272448, 61302028), STMSP Project (no. 2012RZ0005), and the Foundation of Sichuan University Early Career Researcher Award (nos. 2012SCU11036, 2012SCU11070). The authors also acknowledge Dr. Yinjie Lei's help to improve the language expression of this paper.

\section{References}

[1] T. Li, X. Li, J. Wang et al., "Nonlinear sinogram smoothing for low-dose X-ray CT," IEEE Transactions on Nuclear Science, vol. 51, no. 5, pp. 2505-2513, 2004.

[2] J. Xu and B. M. Tsui, "Electronic noise modeling in statistical iterative reconstruction," IEEE Transactions on Image Processing, vol. 18, no. 6, pp. 1228-1238, 2009.

[3] J. Ma, J. Huang, Q. Feng et al., "Low-dose computed tomography image restoration using previous normal-dose scan," Medical Physics, vol. 38, no. 10, pp. 5713-5731, 2011.

[4] A. M. Mendrik, E.-J. Vonken, A. Rutten, M. A. Viergever, and B. Van Ginneken, "Noise reduction in computed tomography scans using 3-D anisotropic hybrid diffusion with continuous switch," IEEE Transactions on Medical Imaging, vol. 28, no. 10, pp. 1585-1594, 2009.

[5] Y. Chen, W. Chen, X. Yin et al., "Improving low-dose abdominal CT images by weighted intensity averaging over large-scale neighborhoods," European Journal of Radiology, vol. 80, no. 2, pp. e42-e49, 2011.

[6] Y. Zhang, J. Zhang, and H. Lu, "Statistical sinogram smoothing for low-dose CT with segmentation-based adaptive filtering," IEEE Transactions on Nuclear Science, vol. 57, no. 5, pp. 25872598, 2010.

[7] E. J. Candès, J. Romberg, and T. Tao, "Robust uncertainty principles: exact signal reconstruction from highly incomplete frequency information," IEEE Transactions on Information Theory, vol. 52, no. 2, pp. 489-509, 2006.

[8] D. L. Donoho, "Compressed sensing," IEEE Transactions on Information Theory, vol. 52, no. 4, pp. 1289-1306, 2006.
[9] G.-H. Chen, J. Tang, and S. Leng, "Prior image constrained compressed sensing (PICCS): a method to accurately reconstruct dynamic CT images from highly undersampled projection data sets," Medical Physics, vol. 35, no. 2, pp. 660-663, 2008.

[10] E. A. Rashed and H. Kudo, "Statistical image reconstruction from limited projection data with intensity priors," Physics in Medicine and Biology, vol. 57, no. 7, pp. 2039-2061, 2012.

[11] J. Ma, H. Zhang, Y. Gao et al., "Iterative image reconstruction for cerebral perfusion CT using a pre-contrast scan induced edgepreserving prior," Physics in Medicine and Biology, vol. 57, no. 22, pp. 7519-7542, 2012.

[12] E. Y. Sidky, C.-M. Kao, and X. Pan, "Accurate image reconstruction from few-views and limited-angle data in divergent-beam CT," Journal of X-Ray Science and Technology, vol. 14, no. 2, pp. 119-139, 2006.

[13] E. Y. Sidky and X. Pan, "Image reconstruction in circular conebeam computed tomography by constrained, total-variation minimization," Physics in Medicine and Biology, vol. 53, no. 17, pp. 4777-4807, 2008.

[14] E. Y. Sidky, R. Chartrand, and X. Pan, "Image reconstruction from few views by non-convex optimization," in Proceedings of the IEEE Nuclear Science Symposium and Medical Imaging Conference (NSS-MIC '07), pp. 3526-3530, Honolulu, Hawaii, USA, November 2007.

[15] L. Ritschl, F. Bergner, C. Fleischmann, and M. Kachelrieß, "Improved total variation-based CT image reconstruction applied to clinical data," Physics in Medicine and Biology, vol. 56, no. 6, pp. 1545-1561, 2011.

[16] H. Yu and G. Wang, "A soft-threshold filtering approach for reconstruction from a limited number of projections," Physics in Medicine and Biology, vol. 55, no. 13, pp. 3905-3916, 2010.

[17] Y. Lu, J. Zhao, and G. Wang, "Few-view image reconstruction with dual dictionaries," Physics in Medicine and Biology, vol. 57, no. 1, pp. 173-189, 2012.

[18] X. Jin, L. Li, Z. Chen, L. Zhang, and Y. Xing, "Anisotropic total variation minimization method for limited-angle CT reconstruction," in Developments in X-Ray Tomography VIII, vol. 8506 of Proceedings of SPIE, 2012.

[19] G. T. Herman and R. Davidi, "Image reconstruction from a small number of projections," Inverse Problems, vol. 24, no. 4, Article ID 045011, 2008.

[20] B. Dong, J. Li, and Z. Shen, "X-Ray CT image reconstruction via wavelet frame based regularization and radon domain inpainting," Journal of Scientific Computing, vol. 54, pp. 333-349, 2013.

[21] Y. Zhang, W. H. Zhang, H. Chen, M. L. Yang, T. Y. Li, and J. L. Zhou, "Few-view image reconstruction combining totaln variation and a high-order norm," International Journal of Imaging Systems and Technology, vol. 23, pp. 249-255, 2013.

[22] Y. Zhang, W. H. Zhang, Y. F. Pu et al., "Few-view image reconstruction with fractional-order total variation," in Proceedings of the 12th International Meeting on Fully Three-Dimensional Image Reconstruction in Radiology and Nuclear Medicine, pp. 177-180, Lake Tahoe, Calif, USA, June 2013.

[23] G. Gilboa and S. Osher, "Nonlocal operators with applications to image processing," Multiscale Modeling and Simulation, vol. 7, no. 3, pp. 1005-1028, 2008.

[24] G. Gilboa and S. Osher, "Nonlocal linear image regularization and supervised segmentation," Multiscale Modeling and Simulation, vol. 6, no. 2, pp. 595-630, 2007. 
[25] Y. Lou, X. Zhang, S. Osher, and A. Bertozzi, "Image recovery via nonlocal operators," Journal of Scientific Computing, vol. 42, no. 2, pp. 185-197, 2010.

[26] X. Zhang, M. Burger, X. Bresson, and S. Osher, "Bregmanized nonlocal regularization for deconvolution and sparse reconstruction," SIAM Journal on Imaging Sciences, vol. 3, no. 3, pp. 253-276, 2010.

[27] R. L. Siddon, "Fast calculation of the exact radiological path for a three-dimensional CT array," Medical Physics, vol. 12, no. 2, pp. 252-255, 1985.

[28] Y. Zhang, Y.-F. Pu, W.-H. Zhang et al., "A novel noniterative metal artifact reduction method using coherence transport with fast marching in computed tomography," International Journal of Imaging Systems and Technology, vol. 22, pp. 1-8, 2012.

[29] Z. Wang, A. C. Bovik, H. R. Sheikh, and E. P. Simoncelli, "Image quality assessment: from error visibility to structural similarity," IEEE Transactions on Image Processing, vol. 13, no. 4, pp. 600612, 2004.

[30] D. L. Donoho and J. M. Johnstone, "Ideal spatial adaptation by wavelet shrinkage," Biometrika, vol. 81, no. 3, pp. 425-455, 1994. 

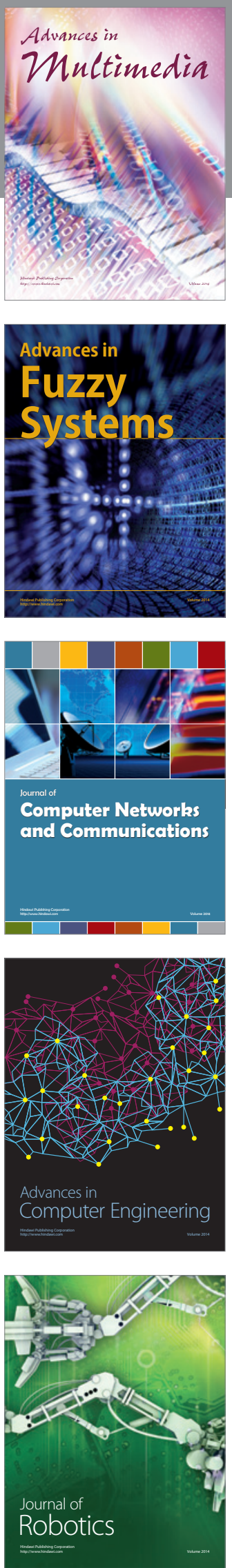

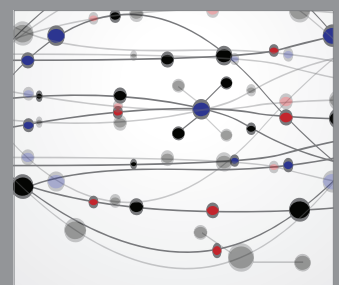

The Scientific World Journal
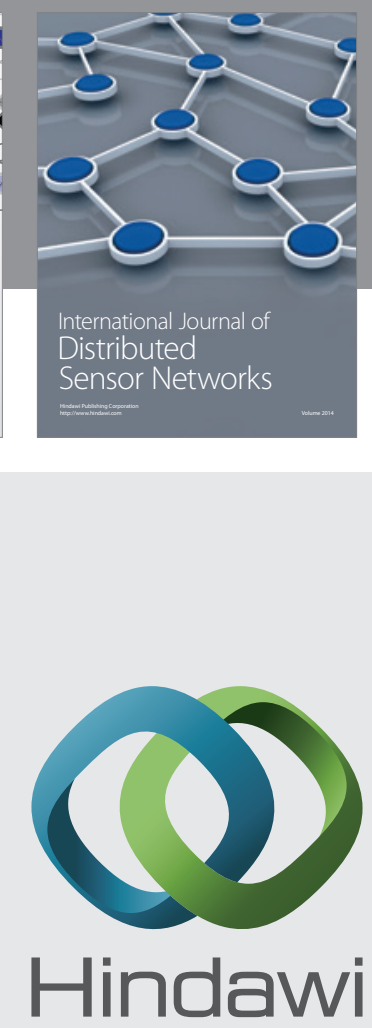

Submit your manuscripts at

http://www.hindawi.com
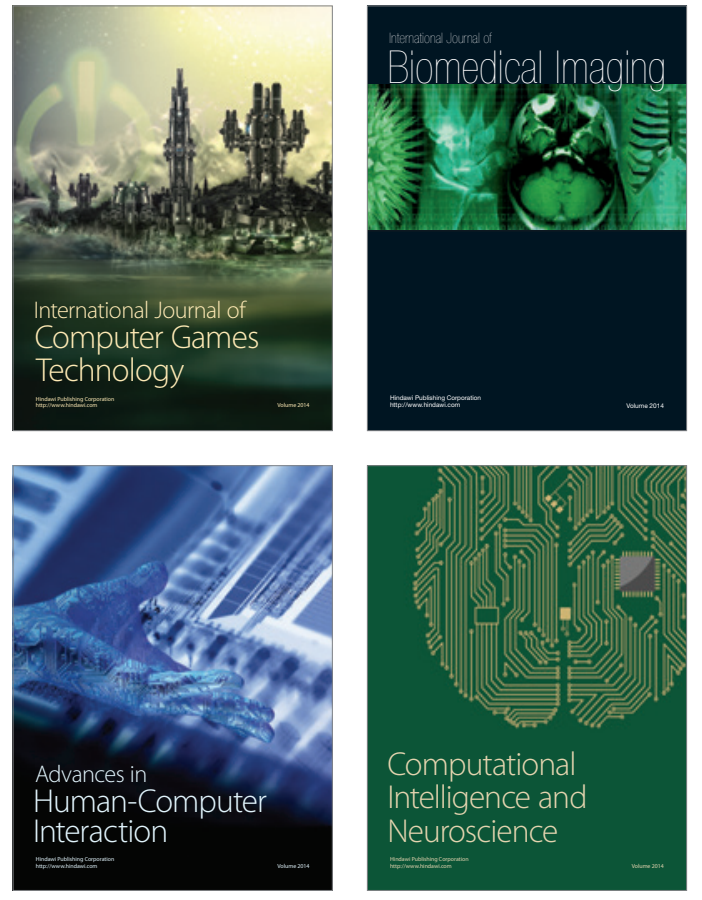
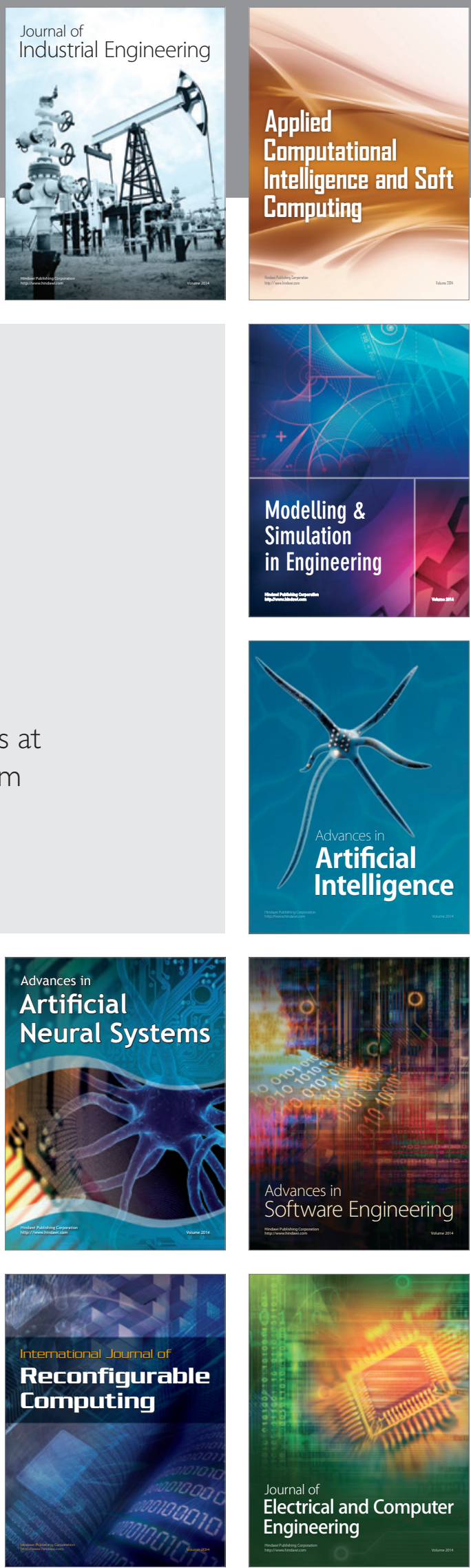